\title{
Ternary Quantized Polar Code Decoders: Analysis and Design
}

\author{
Joachim Neu \\ Stanford University \\ Email: jneu@stanford.edu
}

\author{
Mustafa Cemil Coşkun \\ German Aerospace Center (DLR) \\ Technical University of Munich (TUM) \\ Email: mustafa.coskun@dlr.de
}

\author{
Gianluigi Liva \\ German Aerospace Center (DLR) \\ Email: gianluigi.liva@dlr.de
}

\begin{abstract}
The performance of short polar codes under successive cancellation (SC) and SC list (SCL) decoding is analyzed for the case where the decoder messages are coarsely quantized. This setting is of particular interest for applications requiring lowcomplexity energy-efficient transceivers (e.g., internet-of-things or wireless sensor networks). We focus on the extreme case where the decoder messages are quantized with 3 levels. We show how under SCL decoding quantized log-likelihood ratios lead to a large inaccuracy in the calculation of path metrics, resulting in considerable performance losses with respect to an unquantized SCL decoder. We then introduce two novel techniques which improve the performance of SCL decoding with coarse quantization. The first technique consists of a modification of the final decision step of SCL decoding, where the selected codeword is the one maximizing the maximum-likelihood decoding metric within the final list. The second technique relies on statistical knowledge about the reliability of the bit estimates, obtained through a suitably modified density evolution analysis, to improve the list construction phase, yielding a higher probability of having the transmitted codeword in the list. The effectiveness of the two techniques is demonstrated through simulations.
\end{abstract}

\section{INTRODUCTION}

Following the inclusion of polar codes [1], [2] into the 5th generation cellular communications standard [3], the implementation of efficient polar code decoders has been gaining traction (e.g., [4], [5]). The complexity of a decoder (and hence its power consumption and cost) can be reduced by coarsely quantizing the numeric values stored and processed within the decoder. Simultaneously, however, coarse quantization may deteriorate the decoder's error-correction capability.

To the best of our knowledge, so far the only work to study specifically the effect of coarse quantization on polar decoding is [6]. The authors have chosen 3-level (i.e., ternary) quantization after showing that it is the most extreme viable quantization, i.e., no polarization takes place for 2-level (i.e., binary) quantization. It is furthermore argued that even very coarsely quantized decoding algorithms lead to excellent performance [6] since successive cancellation (SC) decoding achieves a sizeable fraction of capacity for the binary-input additive white Gaussian noise (BiAWGN) channel (cf. Fig.1). When Fig.1 is reparametrized using $\mathrm{E}_{\mathrm{b}} / \mathrm{N}_{0}$ (with $\mathrm{E}_{\mathrm{b}}$ being the energy per information bit and $\mathrm{N}_{0}$ the single-sided noise power spectral density, cf. Fig.2), then a considerable loss in $\mathrm{E}_{\mathrm{b}} / \mathrm{N}_{0}$ occurs due to quantization in the decoder, especially at

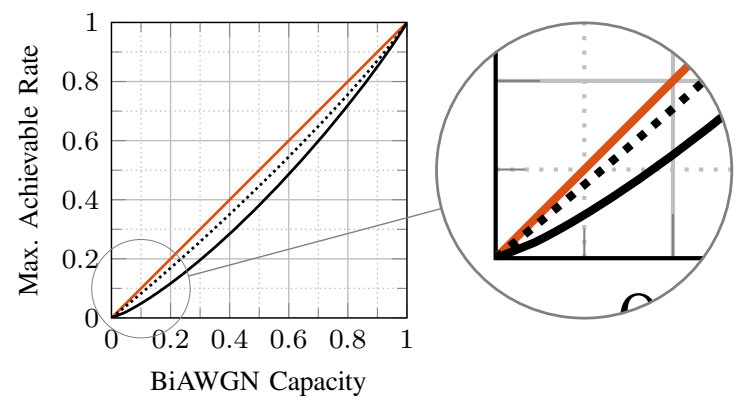

Figure 1. Maximum achievable rate for polar codes vs. capacity of underlying BiAWGN channel; for unquantized SC decoding over unquantized BiAWGN channel $\rightleftharpoons$ and over 3-level quantized BiAWGN (3Q-BiAWGN) channel $\ldots$ and 3-level quantized SC decoding over 3Q-BiAWGN channel

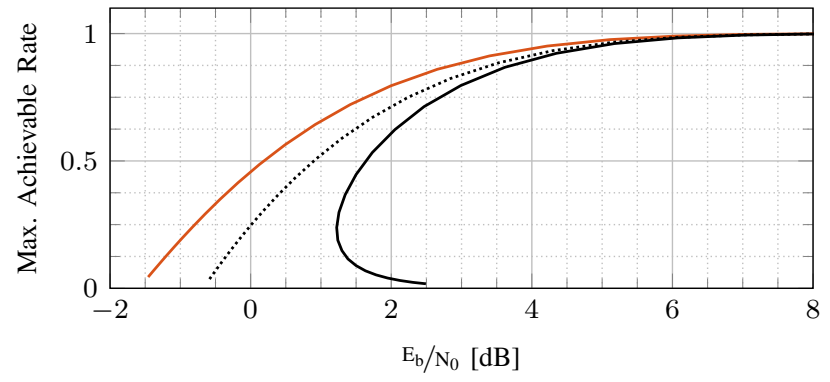

Figure 2. Maximum achievable rate for polar codes vs. $\mathrm{E}_{\mathrm{b}} / \mathrm{N}_{0}$ of underlying BiAWGN channel (same curves $\square \longrightarrow$ and $\square$ as in Fig.11.

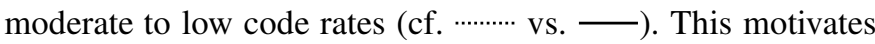
the work on low-complexity techniques to reduce the loss.

We analyze polar code decoding with ternary quantization for short block lengths. In particular, we study the impact of quantization on SC and SC list (SCL) decoding. We show that quantized log-likelihood ratios (LLRs) lead to quantized path metrics (PMs), both of which impair bit estimation and list management of SCL decoders. We devise two novel solutions, namely i) selecting the codeword from the decoder's list based on maximum-likelihood (ML) rather than PM, and ii) utilizing statistical knowledge about the reliability of bit estimates to improve list management. Gains of up to $0.9 \mathrm{~dB}$ in $\mathrm{E}_{\mathrm{b}} / \mathrm{N}_{0}$ at frame error rate (FER) $10^{-3}$ are demonstrated over conventional quantized SCL decoding for a fixed list size. 
In Section【 preliminaries are provided. Then, we present quantized SC and SCL decoders and introduce the first innovation in Section III] In Section IV] our second novel technique is provided. After providing simulation results in Section $\nabla$ the paper concludes with a summary and outlook.

\section{Preliminaries}

\section{A. Notation and Terminology}

Regular and bold letters are used for scalar and vector quantities, respectively, while lowercase and uppercase stand for constants or random variable (RV) realizations and their corresponding RVs, respectively. We use uppercase bold letters for matrix constants (confusion with vector RVs is avoided by context), while calligraphic uppercase letters denote sets. For example, $\lambda$ denotes an LLR value, $\Lambda$ the corresponding random variable, and $\mathcal{L}$ the underlying LLR alphabet. Vectors are column vectors, $\boldsymbol{x} \triangleq\left(x_{0}, \ldots, x_{n-1}\right)^{\top}, \boldsymbol{x}_{i}^{j} \triangleq\left(x_{i}, \ldots, x_{j-1}\right)^{\top}$, and $\boldsymbol{x}^{j} \triangleq \boldsymbol{x}_{0}^{j}$. Probability densities are denoted as $p_{X Y \mid Z}$ or $p(x, y \mid z)$, mass functions as $P_{X Y \mid Z}$ or $P(x, y \mid z)$, the expected value as $\mathrm{E}[X]$. By $\mathrm{C}\left(P_{Y \mid X}\right)$ we denote the capacity of a channel with law $P_{Y \mid X}$.

\section{B. Polar Codes}

Assume $n$ instances of a binary-input symmetric memoryless (BMS) channel $p_{Y \mid X}$ are provided. The polar transform $\boldsymbol{G}_{m}$ is defined [1], [2] as

$$
\boldsymbol{G}_{m} \triangleq \boldsymbol{F}^{\otimes m} \boldsymbol{P}_{m}^{(\text {bitrev })} \quad \text { with } \quad \boldsymbol{F} \triangleq\left[\begin{array}{ll}
1 & 1 \\
0 & 1
\end{array}\right]
$$

where $\boldsymbol{P}_{m}^{(\mathrm{bitrev})}$ is the bit-reversal permutation, and $\boldsymbol{F}^{\otimes m}$ denotes the $m$-fold Kronecker product of $\boldsymbol{F}$. Using $\boldsymbol{G}_{m}$, we obtain $p_{\boldsymbol{Y} \mid \boldsymbol{U}}(\boldsymbol{y} \mid \boldsymbol{u}) \triangleq p_{\boldsymbol{Y} \mid \boldsymbol{X}}\left(\boldsymbol{y} \mid \boldsymbol{G}_{m} \boldsymbol{u}\right)$. Under the assumption of independent and identically distributed (i.i.d.) uniform $U_{i}$, the $i$-th synthetic channel is defined as

$$
p_{\boldsymbol{Y} \boldsymbol{U}^{i} \mid U_{i}}\left(\boldsymbol{y}, \boldsymbol{u}^{i} \mid u_{i}\right) \triangleq \sum_{\boldsymbol{u}_{i+1}^{n} \in\{0,1\}^{n-i-1}} \frac{1}{2^{n-1}} p_{\boldsymbol{Y} \mid \boldsymbol{U}}(\boldsymbol{y} \mid \boldsymbol{u}) \text {. }
$$

A polar code with block length $n \triangleq 2^{m}$ and dimension $k$ is designed by selecting the indices of $k$ synthetic channels into a set $\mathcal{I}$. The $k$ most reliable synthetic channels can be identified, e.g., using density evolution [7]. For encoding, the matrix $\left\{\boldsymbol{G}_{m}\right\}_{\mathcal{I}}$ composed of the columns of $\boldsymbol{G}_{m}$ indexed by the elements of $\mathcal{I}$ is used as generator matrix.

\section{Successive Cancellation Decoding}

The synthetic channels lend themselves to efficient successive decoding, as decoding $u_{0}$ requires only $\boldsymbol{y}$, decoding $u_{1}$ requires $\boldsymbol{y}$ and knowledge of $u_{0}$, decoding $u_{2}$ requires $\left(\boldsymbol{y}, u_{0}, u_{1}\right)$, and so forth. This yields the successive cancellation (SC) decoder. Upon observing channel output $\boldsymbol{y}$, the $i$-th bit $u_{i}$ is estimated from the sign of the corresponding LLR $\lambda_{i}$, defined as

$$
\lambda_{i} \triangleq \log \left(\frac{p_{\boldsymbol{Y} U^{i} \mid U_{i}}\left(\boldsymbol{y}, \hat{\boldsymbol{u}}^{i} \mid 0\right)}{p_{\boldsymbol{Y} U^{i} \mid U_{i}}\left(\boldsymbol{y}, \hat{\boldsymbol{u}}^{i} \mid 1\right)}\right) .
$$

The computations of $\lambda_{i}$ and $u_{i}$ can be defined recursively [7] to achieve an efficient complexity of $O(n \log (n))$.

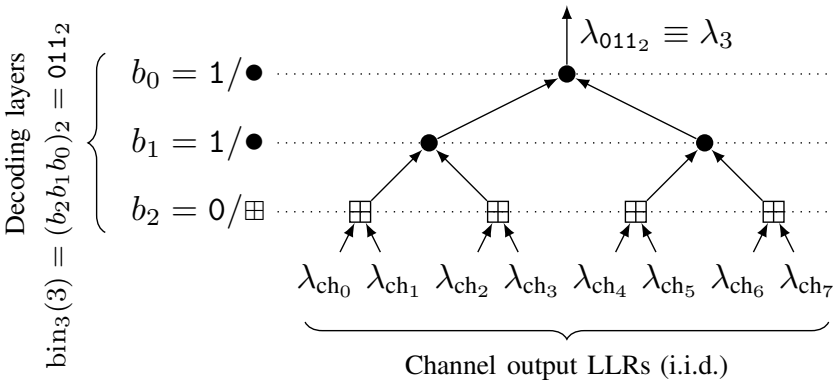

Figure 3. Decoding tree for computation of $\lambda_{011_{2}} \equiv \lambda_{3}$ for $m=3$.

\section{Density Evolution Analysis of SC Decoding}

The performance of SC decoding is analyzed using density evolution. The distribution of $\Lambda_{i}$ is obtained under two assumptions, namely i) the all-zero codeword is transmitted, and ii) the SC decoder is genie-aided, i.e., rather than using $\hat{\boldsymbol{u}}^{i}$ in the computation of $\lambda_{i}$ (eq. (3)), a genie provides $\boldsymbol{u}^{i}$.

The recursive computation of $\lambda_{i}$ is then equivalent to a message-passing procedure over the decoding tree (cf. Fig. 3), constructed as follows: The $2^{m}$ channel output LLRs $\lambda_{\mathrm{ch}_{i}}$ are the leaf nodes of a perfect binary tree of height $m$. The interior nodes are annotated with either $\boxplus$ or $\bullet$ depending on the binary expansion $\operatorname{bin}_{m}(i) \triangleq\left(b_{m-1} \ldots b_{0}\right)_{2} \in\{0,1\}^{m}$ of $i$ of length $m$, with $b_{0}$ being the least significant bit. An interior node of depth $d$, which belongs to the $(m-d)$-th decoding layer, is annotated with $\boxplus$ if $b_{d}=0$ and with $\bullet$ if $b_{d}=1$. Each interior node applies the variable $(\bullet)$ or check node $(\boxplus)$ operation on the two incoming LLR messages, where

$$
\begin{aligned}
x_{1} \bullet x_{2} & \triangleq x_{1}+x_{2}, \\
x_{1} \boxplus x_{2} & \triangleq 2 \tanh ^{-1}\left(\tanh \left(\frac{x_{1}}{2}\right) \tanh \left(\frac{x_{2}}{2}\right)\right) \\
& \approx \operatorname{sign}\left(x_{1}\right) \operatorname{sign}\left(x_{2}\right) \min \left\{\left|x_{1}\right|,\left|x_{2}\right|\right\}
\end{aligned}
$$

and passes its result upwards. The root node's output is $\lambda_{i}$. The so-called min-approximation (eq. (6)) reduces computational complexity with a very limited performance loss [8]. Note that as $\Lambda_{\mathrm{ch}_{i}}$ are i.i.d., so are the messages output by each decoding layer. As a result, $P_{\Lambda_{i}}$ is obtained via density evolution analysis [7].

\section{E. Successive Cancellation List Decoding}

In SCL decoding [9], [10], several instances of an SC decoder are run in parallel, each for a different hypothesis on the past bit decisions. A vector containing the past bit decisions identifies a so-called decoding path. Each path is associated with an index $\ell$ and a path metric (PM) $\mathrm{PM}_{\ell}$. The SCL decoder starts out with one SC instance corresponding to the empty path, as there are no previous bit decisions. The empty path has $\mathrm{PM}_{\emptyset} \triangleq 0$. For each bit $u_{i}, 0 \leq i<n$, and each path $\ell$, the decoder computes $\lambda_{\ell, i}$ and produces the path's two possible offsprings $\ell_{0}$ and $\ell_{1}$ corresponding to $\hat{u}_{i}=0$ and $\hat{u}_{i}=1$, respectively. (If $i \notin \mathcal{I}$, only $\ell_{0}$ is produced.) Their respective $\mathrm{PM}_{\ell_{0}}$ and $\mathrm{PM}_{\ell_{1}}$ are

$$
\mathrm{PM}_{\ell_{u}} \triangleq f_{\mathrm{PMU}}\left(\mathrm{PM}_{\ell}, \lambda_{\ell, i}, u\right) \quad \forall u \in\{0,1\}
$$


where the PM update function $f_{\mathrm{PMU}}(\mathrm{PM}, \lambda, u)$ is defined as

$$
f_{\mathrm{PMU}}(\mathrm{PM}, \lambda, u) \triangleq \mathrm{PM}+\log \left(1+\exp \left((-1)^{1-u} \lambda\right)\right) \text {. }
$$

To mitigate computational complexity, only the $L$ paths with lowest PM are retained at any point, where $L$ is the list size. Upon completion, the collection of paths $\hat{\boldsymbol{u}}_{\ell}$ and their corresponding codewords $\hat{\boldsymbol{c}}_{\ell}$ is called the final list, denoted by $\mathcal{C}_{\text {list }}$. The SCL decoder ultimately decides for the path with lowest PM. For each element $\hat{\boldsymbol{u}}_{\ell}$ in $\mathcal{C}_{\text {list }}$, its $\mathrm{PM}_{\ell}$ is related to its likelihood [10, eq. (13)], i.e.,

$$
\operatorname{PM}_{\ell}=-\log \left(\operatorname{Pr}\left[\boldsymbol{U}=\hat{\boldsymbol{u}}_{\ell} \mid \boldsymbol{Y}=\boldsymbol{y}\right]\right) \text {. }
$$

Hence, selecting the codeword with lowest PM from $\mathcal{C}_{\text {list }}$ is equivalent to taking an ML decision within the list. This holds true for the unquantized SCL decoder. However, as we will see in Section III-B, this is not necessarily true when the LLRs processed within the decoder are quantized. A lower bound on the block error probability of ML decoding (referred to as ML-LB) of a polar code can be estimated via Monte Carlo simulation of SCL decoding by artificially adding the transmitted codeword to $\mathcal{C}_{\text {list }}$ before taking a decision [9].

Since the relation between PM and likelihood of a path does not hold for quantized SCL decoders, we use different frame error rate (FER) definitions as metrics for list decoding: PMFER refers to the FER of an SCL decoder which uses PM to select the codeword from its final list. In contrast, we write LML-FER when ML is used as selection criterion. Finally, List-FER is the list error rate, where a list error is declared whenever the transmitted codeword is not in the final list.

\section{F. Three-Level Quantized BiAWGN Channels}

Consider a binary-input additive white Gaussian noise (BiAWGN) channel with input alphabet $\mathcal{X}=\{-1,+1\}$ and noise variance $\sigma^{2}$ per real-valued signal dimension. The signal-tonoise ratio $(\mathrm{SNR})$ is $\mathrm{E}_{\mathrm{s}} / \mathrm{N}_{0} \triangleq \frac{1}{2 \sigma^{2}}$, where $\mathrm{E}_{\mathrm{S}}$ is the energy per codeword symbol. Furthermore, given a code of rate $R$, $\mathrm{E}_{\mathrm{b}} \triangleq \frac{1}{R} \mathrm{E}_{\mathrm{s}}$. Codeword bits are mapped to channel inputs $0 \mapsto+1,1 \mapsto-1$. For channel output $y$, the channel LLR of the BiAWGN is $\lambda_{\text {ch }}^{\text {(unq) }} \triangleq \frac{2}{\sigma^{2}} y$.

In a 3 -level quantized BiAWGN (3Q-BiAWGN), the channel LLRs $\lambda_{\mathrm{ch}}^{\text {(unq) }}$ are quantized to

$$
\lambda_{\mathrm{ch}}^{(\mathrm{q})} \triangleq \begin{cases}-1 & \text { if } \lambda_{\mathrm{ch}}^{\text {(unq) }} \leq-\delta \\ 0 & \text { if }-\delta<\lambda_{\mathrm{ch}}^{\text {(unq) }}<+\delta \\ +1 & \text { if }+\delta \leq \lambda_{\mathrm{ch}}^{\text {(unq) }}\end{cases}
$$

with reconstruction values $-1 \mapsto-\Delta, 0 \mapsto 0,+1 \mapsto+\Delta$. The channel from $X$ to $\Lambda_{\text {ch }}^{(\mathrm{q})}$ can be seen as a binary error and erasure channel (BEEC). The quantization threshold $\delta$ is chosen to maximize the capacity of the BEEC. When $\lambda_{\mathrm{ch}}^{(\mathrm{q})}$ is input to an unquantized decoder, we choose $\Delta$ to match the LLRs for the BEEC. When $\lambda_{\mathrm{ch}}^{(\mathrm{q})}$ is input to a quantized decoder, we choose $\Delta \triangleq 1$. Experiments corroborate robustness of decoder performance to this choice 1

\footnotetext{
${ }^{1}$ The choice of $\Delta$ does not affect quantized SC decoding, but it does, in principle, affect quantized SCL decoding in the PM update step (eq. (8)). EPMU-enhanced SCL decoding (devised in SectionIV] is not affected.
}

Table I

Check Node (A) and Variable node (B) Operations in $\mathcal{L}_{3}$

\begin{tabular}{r|rrr}
\hline$\boxplus$ & -1 & 0 & +1 \\
\hline-1 & +1 & 0 & -1 \\
0 & 0 & 0 & 0 \\
+1 & -1 & 0 & +1 \\
\hline
\end{tabular}

(a) Check node operation $\boxplus$

\begin{tabular}{r|rrr}
\hline \multicolumn{1}{c|}{$\bullet$} & -1 & 0 & +1 \\
\hline-1 & -1 & -1 & 0 \\
0 & -1 & 0 & +1 \\
+1 & 0 & +1 & +1 \\
\hline
\end{tabular}

(b) Variable node operation $\bullet$

\section{Quantized Polar Code Decoding}

\section{A. Quantized SC Decoding}

In Section II-C we revisit how SC decoding is viewed as a message-passing procedure over trees (cf. Fig.3). This is a natural junction at which to separate the SC decoding algorithm (i.e., the sequence of operations) from the specifics of the underlying LLR algebra $(\mathcal{L}, \boxplus, \bullet,-)$, which is a set of possible LLR values $\mathcal{L}$ with operations $\boxplus, \bullet: \mathcal{L} \times \mathcal{L} \rightarrow \mathcal{L}$ and - : $\mathcal{L} \rightarrow \mathcal{L}$. Given any LLR algebra $\mathcal{L}$, an $\mathcal{L}$-SC decoder and a corresponding density evolution are readily instantiated. This abstraction provides a framework to analyze 'plain' quantized $\mathrm{SC}(\mathrm{L})$ decoders and more involved constructions in Section IV]

The 3-level quantized $\mathcal{L}_{3}$-SC decoder uses $\mathcal{L}_{3} \triangleq\{0, \pm 1\}$, with operations defined analogous to the min-sum rules (eqs (4) and (6)), but clipped to $\mathcal{L}_{3}$ (cf. Table I). We refer to the unquantized $\mathrm{SC}$ decoder as $\mathcal{L}_{\infty}$-SC decoder.

We compare $\mathcal{L}_{\infty}$-SC decoding for BiAWGN and 3Q-BiAWGN with $\mathcal{L}_{3}$-SC decoding for 3 Q-BiAWGN. Polar codes with $n \in\{128,256\}$ and $R=1 / 2$ were designed using density evolution [7]. At FER $10^{-3}$, a loss of $0.8 \mathrm{~dB}$ in $\mathrm{E}_{\mathrm{b}} / \mathrm{N}_{0}$ is caused by channel output quantization, and a further loss of $1.2 \mathrm{~dB}$ in $\mathrm{E}_{\mathrm{b}} / \mathrm{N}_{0}$ is caused by quantized decoding. These losses are in the range predicted by previous asymptotic analysis (cf. Fig.2).

\section{B. Quantized SCL Decoding}

We extend the $\mathcal{L}$-SC and SCL into the $\mathcal{L}$-SCL decoder. In SCL decoding, the PM update is approximated [10, eq. (12)]

$$
f_{\mathrm{PMU}}(\mathrm{PM}, \lambda, u) \approx \mathrm{PM}+\max \left\{0,(-1)^{1-u} \lambda\right\} .
$$

This approximation is not suited for quantized decoding, e.g., for $\mathcal{L}_{3}$ and $u=0$ it maps both $\lambda \in\{0,+1\}$ to the same PM update. We therefore use the refinement [11, eq. (3.8)],

$$
\begin{aligned}
& f_{\mathrm{PMU}}(\mathrm{PM}, \lambda, u) \approx \tilde{f}_{\mathrm{PMU}}(\mathrm{PM}, \lambda, u) \triangleq \\
& \mathrm{PM}+ \begin{cases}(-1)^{1-u} \lambda & \text { if }(-1)^{u} \lambda<-2 \ln (2) \\
\frac{1}{2}(-1)^{1-u} \lambda+\ln (2) & \text { if }|\lambda| \leq 2 \ln (2) \\
0 & \text { if }(-1)^{u} \lambda>+2 \ln (2) .\end{cases}
\end{aligned}
$$

We use the reconstruction values as $\lambda$ for quantized LLRs. As for the unquantized SCL decoder in Section【-E the conventional quantized $\mathcal{L}$-SCL decoder selects the path with lowest PM from its final list $\mathcal{C}_{\text {list }}$.

Note that quantized LLRs undergo severe distortion due to rounding and clipping. This carries over to PMs. PMs computed from imprecise LLRs do not preserve the order in likelihood among paths and become de-facto quantized. Both effects render PMs little useful for selecting a path from $\mathcal{C}_{\text {list }}$. 
We compare $\mathcal{L}_{\infty}$-SCL for BiAWGN and 3Q-BiAWGN with $\mathcal{L}_{3}$-SCL for 3Q-BiAWGN using the codes from Section varying $L \in\{1,32,128\}$. As expected, $\mathcal{L}_{3}$-SCL decoding improves over $\mathcal{L}_{3}$-SC decoding, e.g., $0.8 \mathrm{~dB}$ in $\mathrm{E}_{\mathrm{b}} / \mathrm{N}_{0}$ at FER $10^{-3}$ for $n=256$ and $R=1 / 2$. But the same gains hold for $\mathcal{L}_{\infty}$-SCL vs. $\mathcal{L}_{\infty}$-SC over $3 \mathrm{Q}$-BiAWGN, so that the gap due to quantization in the decoder remains unaltered. There are considerable gaps between List-FER and PM-FER of $\mathcal{L}_{3}$ SCL, which suggests that often the transmitted codeword is contained in SCL's final list but not selected according to PM.

\section{Quantized SCL Decoding with In-List ML}

A final list $\mathcal{C}_{\text {list }}$ is formed via the $\mathcal{L}$-SCL decoding procedure as in Section $\amalg I-B$ Within the list $\mathcal{C}_{\text {list }}$ of candidate codewords, the ML rule is applied to select the most likely codeword, i.e.,

$$
\hat{\boldsymbol{c}}_{\mathrm{ML}}=\underset{\boldsymbol{c} \in \mathcal{C}_{\text {list }}}{\arg \max } P(\boldsymbol{y} \mid \boldsymbol{c}) .
$$

Simulations show that in-list ML reliably achieves either i) the PM-FER of $\mathcal{L}_{\infty}$-SCL, or ii) the List-FER of $\mathcal{L}_{3}$-SCL (whichever is worse, both over 3Q-BiAWGN). For i), no improvement can be expected as $\mathcal{L}_{\infty}$-SCL tightly matches the ML-LB. But then, ii) suggests that quantization causes the transmitted codeword to often be inadvertently removed from the list during decoding. This prompts the development of techniques to enhance list management in SCL in Section[IV

\section{List Enhancement Techniques}

We use statistical knowledge about the LLRs computed during unquantized decoding to modify the behavior of a quantized decoder in such a way that it mimics the behavior of the unquantized decoder as closely as possible, given only the instantaneous information contained in its quantized LLRs. In particular, we modify the quantized decoder to emulate the PM update step of an unquantized decoder.

Let $f_{\Delta \mathrm{PM}}^{(i)}\left(\lambda_{i}, u_{i}\right)$ be the PM increment of a path $\ell_{u_{i}}$ that computed LLR $\lambda_{i}$ and decided for bit $u_{i}$. In an $\mathcal{L}_{3}$-SCL decoder, $f_{\triangle \mathrm{PM}}^{(i)}$ only encounters six different input combinations, $\mathcal{L} \times\{0,1\}$. Rather than computing $f_{\triangle \mathrm{PM}}^{(i)}$ using eq. (13) and the reconstruction value associated with $\lambda_{i}$, a lookup table

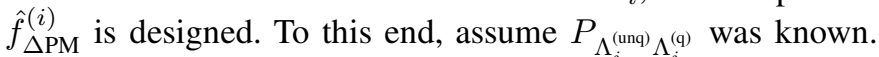
Then, the mean squared error between the $\mathrm{PM}$ updates in the unquantized and the quantized decoder is minimized with

$$
\hat{f}_{\Delta \mathrm{PM}}^{(i)}\left(\lambda_{i}^{(\mathrm{q})}, u_{i}\right) \triangleq \mathrm{E}\left[f_{\Delta \mathrm{PM}}^{(i)}\left(\Lambda_{i}^{(\mathrm{unq})}, u_{i}\right) \mid \Lambda_{i}^{(\mathrm{q})}=\lambda_{i}^{(\mathrm{q})}\right] .
$$

Hence the name expected path metric updates (EPMU).

The remainder of this section describes a construction for

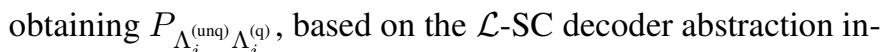
troduced in Section [II-A To this end, imagine a joint decoder composed of an unquantized and a quantized decoder (cf. Fig.(4). Both decoders operate on the same channel realization, i.e., the $\Lambda_{\mathrm{ch}_{i}}^{(\mathrm{q})}$ and $\Lambda_{\mathrm{ch}_{i}}^{(\mathrm{unq})}$ are not independent. The output for the $i$-th synthetic channel of the joint decoder is $\left(\Lambda_{i}^{(\text {unq) }}, \Lambda_{i}^{(\mathrm{q})}\right)$. A joint density evolution, under the assumptions of all-zero transmitted codeword and genie-aided SC decoding, as in

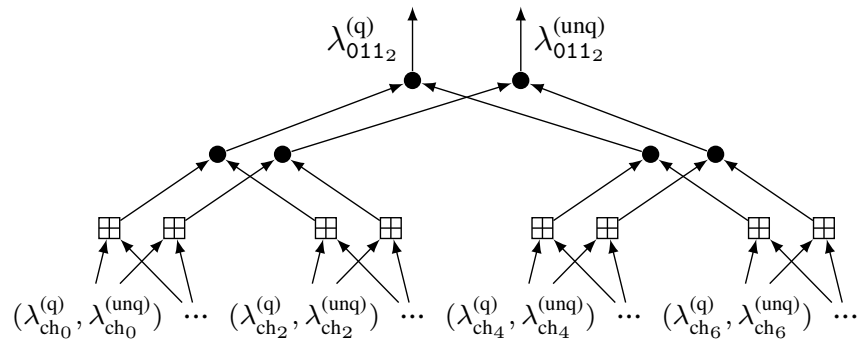

Figure 4. Coupling of a quantized and an unquantized decoder, which compute LLRs $\lambda_{i}^{\text {(q) }}$ and $\lambda_{i}^{\text {(unq) }}$, respectively, in parallel but based on the same channel realization (i.e., the $\Lambda_{\mathrm{ch}_{i}}^{(\mathrm{q})}$ and $\Lambda_{\mathrm{ch}_{i}}^{(\mathrm{unq})}$ are not independent).

Section $[I-D$, is used to analyze the joint decoder in order to obtain $P_{\Lambda_{i}^{(\mathrm{unq})}} \Lambda_{i}^{(\mathrm{q})}$.

Note that the joint decoder depicted in Fig. 4 can be viewed as a $\mathcal{L}_{(3, \infty)}$-SC decoder with $\mathcal{L}_{(3, \infty)} \triangleq \mathcal{L}_{3} \times \mathcal{L}_{\infty}$, where the LLR operations are reduced to those of the underlying quantized and unquantized decoder, i.e.,

$$
\begin{aligned}
\left(x_{1}^{(\mathrm{q})}, x_{1}^{(\text {unq })}\right) \boxplus\left(x_{2}^{(\mathrm{q})}, x_{2}^{(\text {unq })}\right) \triangleq\left(x_{1}^{(\mathrm{q})} \boxplus x_{2}^{(\mathrm{q})}, x_{1}^{(\text {unq })} \boxplus x_{2}^{(\text {unq })}\right) \\
\left(x_{1}^{(\mathrm{q})}, x_{1}^{\text {(unq) })} \bullet\left(x_{2}^{(\mathrm{q})}, x_{2}^{\text {(unq) }}\right) \triangleq\left(x_{1}^{(\mathrm{q})} \bullet x_{2}^{(\mathrm{q})}, x_{1}^{\text {(unq) }} \bullet x_{2}^{(\text {unq })}\right)\right. \\
-\left(x^{(\mathrm{q})}, x^{\text {(unq) })} \triangleq\left(-x^{(\mathrm{q})},-x^{(\text {unq })}\right) .\right.
\end{aligned}
$$

Furthermore, recall that under the all-zero transmitted codeword assumption, the BiAWGN channel output $Y$ is Gaussian, $Y \sim \mathcal{N}_{\mathbb{R}}\left(1, \sigma^{2}\right)$. Hence, for $\mu \triangleq \frac{2}{\sigma^{2}}, \Lambda_{\mathrm{ch}}^{\text {(unq) }} \sim \mathcal{N}_{\mathbb{R}}(\mu, 2 \mu)$. When $\lambda_{\mathrm{ch}}^{\text {(unq) }}$ and $\lambda_{\mathrm{ch}}^{(\mathrm{q})}$ are computed from the same channel realization $y$, as is the case for the joint decoder at hand, only tuples $\left(\lambda_{\text {ch }}^{(\mathrm{q})}, \lambda_{\text {ch }}^{(\text {unq) }}\right) \in \mathcal{L}^{\prime} \triangleq \mathcal{L}_{-1}^{\prime} \cup \mathcal{L}_{0}^{\prime} \cup \mathcal{L}_{+1}^{\prime}$ can occur, with

$$
\begin{aligned}
\mathcal{L}^{\prime}{ }_{-1} & \triangleq(-\infty,-\delta] \times\{-1\} \\
\mathcal{L}_{0}^{\prime} & \triangleq(-\delta,+\delta) \times\{0\} \\
\mathcal{L}_{+1}^{\prime} & \triangleq[+\delta,+\infty) \times\{+1\} .
\end{aligned}
$$

Then,

$P\left(\lambda_{\mathrm{ch}}^{(\mathrm{q})}, \lambda_{\mathrm{ch}}^{\text {(unq) }}\right)= \begin{cases}\phi_{(\mu, 2 \mu)}\left(\lambda_{\mathrm{ch}}^{\text {(unq) }}\right) & \text { if }\left(\lambda_{\mathrm{ch}}^{(\mathrm{q})}, \lambda_{\mathrm{ch}}^{\text {(unq) }}\right) \in \mathcal{L}^{\prime} \\ 0 & \text { otherwise }\end{cases}$

where $\phi_{\left(\mu, \sigma^{2}\right)}(x) \triangleq \frac{1}{\sqrt{2 \pi \sigma^{2}}} \exp \left(-\frac{(x-\mu)^{2}}{2 \sigma^{2}}\right)$. Obviously, $\Lambda_{\mathrm{ch}}^{(\mathrm{q})}$ and $\Lambda_{\mathrm{ch}}^{\text {(unq) }}$ are not independent.

Density evolution is carried out on the $\mathcal{L}_{(3, \infty)}$-SC decoder to obtain $\tilde{P}_{\Lambda_{i}^{\text {(un) })} \Lambda_{i}^{(\mathrm{q})}}$, the distribution of $\boldsymbol{\Lambda}_{i} \triangleq\left(\Lambda_{i}^{\text {(unq) }}, \Lambda_{i}^{(\mathrm{q})}\right)$ conditional on the all-zero codeword assumption. The unconditional distribution is then obtained by symmetry as

$$
P_{\boldsymbol{\Lambda}_{i}}\left(\boldsymbol{\lambda}_{i}\right) \triangleq \begin{cases}\frac{1}{2} \tilde{P}_{\boldsymbol{\Lambda}_{i}}\left(\boldsymbol{\lambda}_{i}\right)+\frac{1}{2} \tilde{P}_{\boldsymbol{\Lambda}_{i}}\left(-\boldsymbol{\lambda}_{i}\right) & \text { if } i \in \mathcal{I} \\ \tilde{P}_{\boldsymbol{\Lambda}_{i}}\left(\boldsymbol{\lambda}_{i}\right) & \text { otherwise. }\end{cases}
$$

EPMUs are designed from $P_{\Lambda_{i}^{(\text {unq })} \Lambda_{i}^{(q)}}$ using eq. (15) 2

\footnotetext{
${ }^{2}$ Using similar joint density evolution analyses, extensions of EPMU can be implemented, e.g., the number of contradictions encountered at variable nodes or the number of double erasures encountered at check nodes can serve as a low-complexity reliability indicator to further refine EPMUs [11 Sec. 4.2].
} 


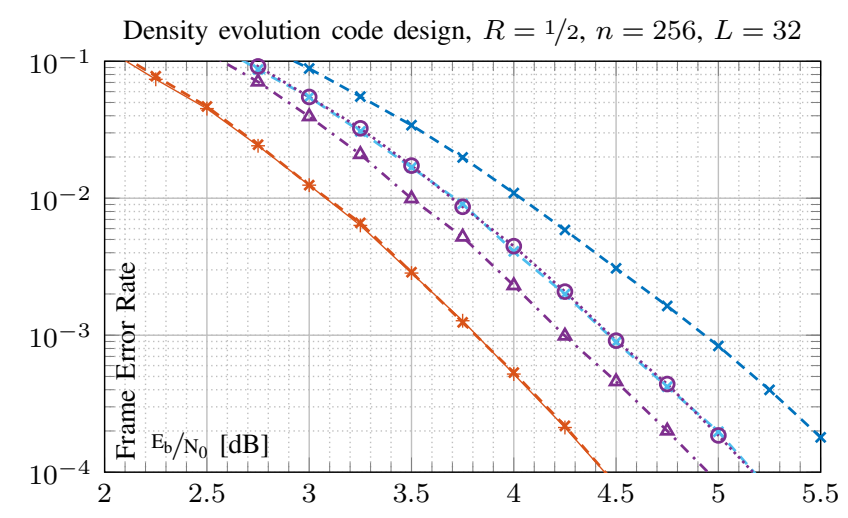

(a) FER vs. $\mathrm{E}_{\mathrm{b}} / \mathrm{N}_{0}$ for variants of SCL decoding over the 3Q-BiAWGN channel, demonstrating gains for in-list ML and EPMU.

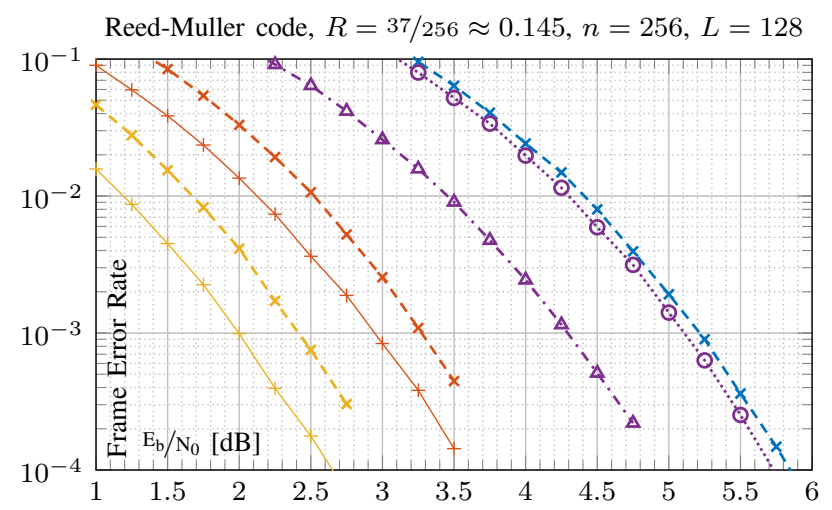

(b) FER vs. $\mathrm{E}_{\mathrm{b}} / \mathrm{N}_{0}$ for variants of SCL decoding over the 3Q-BiAWGN channel, underlining the utility of EPMU at low code rates.

\begin{tabular}{|c|c|c|c|}
\hline & CHANNEL & DECODER & METRIC \\
\hline$-*---$ & 3Q-BiAWGN & $\mathcal{L}_{3}-\mathrm{SCL}$ & PM-FER \\
\hline$--x-\theta$ & 3Q-BiAWGN & $\mathcal{L}_{3}-\mathrm{SCL}$ & List-FER \\
\hline$\cdots \theta \cdots$ & 3Q-BiAWGN & $\mathcal{L}_{3}-\mathrm{SCL}+$ in-list $\mathrm{ML}$ & LML-FER \\
\hline$-\cdot \Delta \cdot \cdot$ & 3Q-BiAWGN & $\mathcal{L}_{3}$-SCL + in-list $\mathrm{ML}+\mathrm{EPMU}$ & LML-FER \\
\hline$--x-4$ & 3Q-BiAWGN & $\mathcal{L}_{\infty}-\mathrm{SCL}$ & PM-FER \\
\hline+1 & 3Q-BiAWGN & $\mathcal{L}_{\infty}-\mathrm{SCL}$ & ML-LB \\
\hline$-x-=$ & BiAWGN & $\mathcal{L}_{\infty}-\mathrm{SCL}$ & PM-FER \\
\hline$\square$ & BiAWGN & $\mathcal{L}_{\infty}^{\infty}-\mathrm{SCL}$ & ML-LB \\
\hline
\end{tabular}

Figure 5. FER vs. $\mathrm{E}_{\mathrm{b}} / \mathrm{N}_{0}$, (a) $R=1 / 2$ Polar code, and (b) $R=37 / 256$ Reed-Muller code, demonstrating the gains due to the proposed techniques.

\section{Simulation Results}

Simulations demonstrate that the proposed techniques boost quantized polar code decoding across a wide range of scenarios. Our figure of merit is $\mathrm{E}_{\mathrm{b}} / \mathrm{N}_{0}$ at a target FER of $10^{-3}$.

\section{A. Rate $1 / 2$}

In Fig.5(a), we consider the codes from Sections III-A and III-B with $R=1 / 2, n=256$ and $L=32$. In-list ML $\mathcal{L}_{3}$-SCL gains $0.5 \mathrm{~dB}$ over conventional $\mathcal{L}_{3}$-SCL and tightly

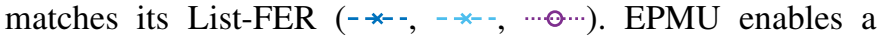
further gain of $0.2 \mathrm{~dB}$ by improving the List-FER (…… Overall, $0.7 \mathrm{~dB}$ of the $1.2 \mathrm{~dB}$ losses due to quantization in the conventional decoder are reclaimed using the proposed low

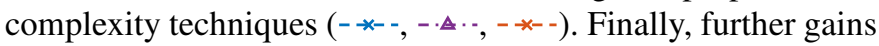
can be achieved by increasing $L$ (e.g., for $L=128$, the gap from $\mathcal{L}_{3}$-SCL with in-list ML and EPMU to the ML-LB of $\mathcal{L}_{\infty}-\mathrm{SCL}$ reduces to $0.2 \mathrm{~dB}$ [11, Fig. 4.8]).

\section{B. Low Code Rate}

In Fig.5(b) we consider the Reed-Muller code with $n=$ 256 and $R=37 / 256 \approx 0.145$, which is in the low rate regime where pronounced losses due to quantization are expected (cf. Fig.22). At $L=128$, the losses amount to $1.9 \mathrm{~dB}\left(-x^{-x}, E^{-x--}\right)$. While in-list ML alone brings no considerable gain $1-*--$

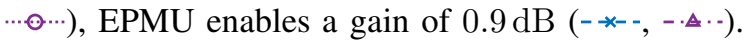

\section{CONCLUSION}

We analyzed the effects of coarse quantization on SC and SCL decoding of polar codes with short block lengths. Quantized LLRs lead to quantized PMs, both of which impair bit estimation and list management. We demonstrated that in-list ML and EPMU can overcome these impairments, providing gains of up to $0.9 \mathrm{~dB}$ in $\mathrm{E}_{\mathrm{b}} / \mathrm{N}_{0}$ at FER $10^{-3}$ over conventional quantized SCL decoding for the provided examples.

\section{ACKNOWLEDGEMENTS}

The authors thank Hamed Hassani, Gerhard Kramer and Rüdiger Urbanke for helpful suggestions and fruitful discussions. The work of J. Neu was conducted in part during a Summer@EPFL research fellowship (https://summer.epfl.ch/). The work of M. Coşkun was supported by the research grant "Efficient Coding and Modulation for Satellite Links with Severe Delay Constraints" funded by Munich Aerospace e.V.

\section{REFERENCES}

[1] N. Stolte, "Rekursive Codes mit der Plotkin-Konstruktion und ihre Decodierung," Ph.D. dissertation, Technische Universität Darmstadt, 2002.

[2] E. Arıkan, "Channel polarization: A method for constructing capacityachieving codes for symmetric binary-input memoryless channels," IEEE Trans. Inf. Theory, vol. 55, no. 7, pp. 3051-3073, Jul. 2009.

[3] 3GPP, "NR; Multiplexing and channel coding," 3rd Generation Partnership Project (3GPP), Technical Specification 38.212, 2018, version 15.0.0.

[4] P. Giard, G. Sarkis, A. Balatsoukas-Stimming, Y. Fan, C. Tsui, A. Burg, C. Thibeault, and W. J. Gross, "Hardware decoders for polar codes: An overview," in Proc. IEEE Int. Symp. Circuits Syst. (ISCAS), Montreal, QC, Canada, May 2016, pp. 149-152.

[5] P. Giard, A. Balatsoukas-Stimming, T. C. Müller, A. Bonetti, C. Thibeault, W. J. Gross, P. Flatresse, and A. Burg, "PolarBear: A 28-nm FD-SOI ASIC for decoding of polar codes," IEEE J. Emerg. Sel. Topics Circuits Syst., vol. 7, no. 4, pp. 616-629, Dec. 2017.

[6] S. H. Hassani and R. Urbanke, "Polar codes: Robustness of the successive cancellation decoder with respect to quantization," in Proc. IEEE Int. Symp. Inf. Theory (ISIT), Cambridge, MA, USA, Jul. 2012, pp. 1962-1966.

[7] R. Mori and T. Tanaka, "Performance and construction of polar codes on symmetric binary-input memoryless channels," in Proc. IEEE Int. Symp. Inf. Theory (ISIT), Seoul, South Korea, Jun. 2009, pp. 1496-1500.

[8] C. Leroux, I. Tal, A. Vardy, and W. J. Gross, "Hardware architectures for successive cancellation decoding of polar codes," in Proc. IEEE Int. Conf. Acoustics, Speech, Signal Process. (ICASSP), Prague, Czech Republic, May 2011, pp. 1665-1668.

[9] I. Tal and A. Vardy, "List decoding of polar codes," IEEE Trans. Inf. Theory, vol. 61, no. 5, pp. 2213-2226, May 2015.

[10] A. Balatsoukas-Stimming, M. B. Parizi, and A. Burg, "LLR-based successive cancellation list decoding of polar codes," IEEE Trans. Signal Process., vol. 63, no. 19, pp. 5165-5179, Oct. 2015.

[11] J. Neu, "Quantized polar code decoders: Analysis and de sign," Master's thesis, Technical University of Munich, 2018, arXiv:1902.10395v1 [cs.IT] 